Artigo Original

\title{
Visibilidade dos braços afeta a preferência manual em bebês
}

\author{
Lívia Silveira Pogetti ${ }^{1,2}$ \\ Rosana Machado de Souza ${ }^{1,2}$ \\ Eloísa Tudella ${ }^{2}$ \\ Luis Augusto Teixeira ${ }^{1}$
${ }^{1}$ Laboratório Sistemas Motores Humanos, Escola de Educação Física e Esporte da Universidade de São Paulo (USP), São Paulo, SP, Brasil
${ }^{2}$ Laboratório de Pesquisa e Análise do Movimento, Departamento de Fisioterapia da Universidade Federal de São Carlos (UFSCar), São Carlos, SP, Brasil

\begin{abstract}
Resumo: Um aspecto de interesse sobre a formação da preferência manual humana em idades precoces é a extensão em que ela é afetada por informações aferentes. O objetivo deste estudo foi investigar o efeito da oclusão visual do braço preferido sobre a preferência manual e desempenho motor em bebês. Participaram cinco bebês com cinco meses de idade, que realizaram alcances com visão plena ou oclusão visual do braço preferido. O desempenho motor foi avaliado por meio de medidas cinemáticas. Os resultados indicaram que a oclusão visual induziu redução da frequência de alcances unimanuais com o braço ocluído durante e imediatamente após a oclusão visual. Oclusão visual não alterou o desempenho motor. Estes resultados indicam que a formação da preferência manual durante o desenvolvimento motor é afetada pela disponibilidade de informação visual dos braços, embora os bebês pareçam ter pouca capacidade de usar a visão para controle motor.
\end{abstract}

Palavras-chave: Manualidade. Lateralidade. Alcance. Visão. Bebês.

\section{Arms visibility affects manual preference in infants}

\begin{abstract}
An interesting aspect about formation of human manual preference in early ages is the extent to which it is affected by afferent information. This study aimed at investigating the effect of visual occlusion of the preferred arm on manual preference and motor performance in infants. Five 5-month-old infants performed reaching movements under full vision or occlusion of their preferred arm. Motor performance was assessed through kinematic measures. Results indicated that visual occlusion led to reduction of frequency of unimanual reaches using the visually occluded arm. Visual occlusion did not impair motor performance. These results indicate that formation of manual preference during motor development is affected by availability of visual afference of the arms, although infants seem to have reduced capacity to use vision for motor control.
\end{abstract}

Keywords: Handedness. Laterality. Reaching. Vision. Infants.

\section{Introdução}

Definida pela maior frequência de uso de uma das mãos para a realização de tarefas manuais (TEIXEIRA, 2006), a preferência manual tem sido concebida como derivada fundamentalmente de fatores genéticos (cf. ANNETT, 1985; LEVY; NAGYLAKI, 1972; MCMANUS, 1985). Essa concepção, entretanto, está em desacordo com evidências indicando que a preferência manual pode ser decorrente de experiências sensóriomotoras vivenciadas em idades precoces (CORBETTA; THELEN, 2002; veja também CORBETTA; WILLIAMS; SNAPP-CHILDS, 2006) ou influenciada por outros fatores epigenéticos, tais como o ambiente (HARKINS; MICHEL, 1988; HARKINS; UZGIRIS, 1991; MANNS, 2006), tipo de tarefa (BRYDEN; MAYER; ROY, 2011; PROVINS, 1997; TEIXEIRA, 2000) e aspectos culturais (ASHTON, 1982). De particular interesse para o presente estudo, foi observado que em crianças de 7 a 11 anos portadoras da síndrome do torcicolo congênito, a preferência manual esteve associada com o lado de inclinação da cabeça (OCKLENBURG et al., 2010). Essa associação tem sido interpretada como sendo consequência do maior contato visual com um dos braços, provocado pela postura da cabeça voltada permanentemente para o lado esquerdo ou direito.

A orientação da cabeça predominantemente para um dos lados do corpo também tem sido observada em bebês com desenvolvimento normal durante o período de manifestação dos reflexos primitivos. Especificamente, tem sido evidenciado um viés lateral no reflexo tônico 
cervical assimétrico, fazendo com que a face fique mais tempo voltada para o lado direito do corpo (TURKEWITZ; BIRCH; MOREAU, 1966; TURKEWITZ et al., 1969; VERVERS et al., 1994; VIVIANI; TURKEWITZ; KARP, 1978), induzindo assim maior contato visual com o braço direito. A observação do braço em movimento mobiliza recursos atencionais (CONTE et al., 2007), e a orientação da face para o lado direito poderia induzir o uso mais frequente do braço desse mesmo lado. A prática sistemática desta ação sensório-motora promove transformações estruturais e funcionais no sistema nervoso específicas ao hemisfério cerebral mais ativo (NUDO et al., 1996; RIDDING; FLAVEL, 2006).Tal fato pode desencadear assimetrias nas redes neurais associadas à preferência manual $e$ diferenças de desempenho motor entre os braços. Na dimensão comportamental, o uso mais frequente de um dos braços e orientação dos movimentos via feedback visual pode resultar em um controle mais refinado deste braço. A partir de movimentos de alcance mais bem controlados com um dos braços, seria estabelecida então uma preferência lateral baseada em efetiva diferença da capacidade de controle entre os membros.

Nota-se, portanto, que assim como para a formação da lateralidade, a informação visual tem sido demonstrada ser um importante recurso no desenvolvimento do controle motor (DARLING; RIZZO, 2001; VAN DER MEER, 1997; $\underline{\text { VAN DER }}$ MEER; VAN DER WEEL, 1995; VON HOFSTEN, 1982), especificamente, na modulação em tempo real dos movimentos dos braços (LASKY, 1977; MCDONNELL, 1979; VON HOFSTEN; FAZELZANDY, 1984).

Em uma perspectiva de desenvolvimento motor, torna-se oportuno destacar que tem sido evidenciado que áreas visuais apresentam um pico no seu desenvolvimento entre as idades de dois e três meses, atingindo a maturidade aos cinco meses (ROSANDER; NYSTRÖM; GREDEBÄCK; VON HOFSTEN, 2007). A partir deste marco desenvolvimental, cinco meses de idade corresponde ao período em que os bebês percebem as informações visuais de forma mais detalhada do ambiente e dos seus movimentos, estando aptos a utilizar a informação visual para o controle motor (CORBETTA; GUAN; WILLIAMS, 2011). Dessa forma, privar a visualização do movimento de um dos braços nesta idade pode promover modificação na preferência do braço para alcançar. A análise dos efeitos da visibilidade dos braços em idades precoces poderia, assim, fornecer informações relevantes sobre o papel da aferência visual no desenvolvimento da preferência manual humana.

O objetivo do presente estudo foi investigar o efeito da oclusão visual na preferência manual de bebês. Especificamente, neste estudo foi avaliado o efeito da oclusão da visibilidade do braço preferido sobre a frequência de seu uso e características cinemáticas no alcance de um objeto estático. A avaliação foi feita antes, durante e após o período de oclusão visual. As hipóteses testadas foram de que a oclusão da visão do braço preferido (1) reduz a frequência de alcances unimanuais com este braço e (2) prejudica o desempenho motor do braço com visibilidade ocluída.

\section{Métodos}

\section{Participantes}

Participaram deste estudo 10 bebês saudáveis de ambos os sexos. Foram excluídos quatro bebês por não comparecimento à avaliação, e um por não se adequar aos critérios de inclusão. Concluíram o experimento cinco bebês com cinco meses de idade, sendo três meninas. Os critérios de inclusão foram os seguintes: bebês saudáveis, com idade gestacional de 37 a 41 semanas $(M=$ $38,8, d p=1,3$ ), índice de Apgar igual ou superior a 8 no primeiro e quinto minutos, classificados como bebês vigorosos que não necessitavam de cuidados especiais. Este estudo foi aprovado pelo Comitê de Ética local.

\section{Tarefa e equipamento}

Os bebês foram posicionados em uma cadeira infantil reclinada em $50^{\circ} \mathrm{em}$ relação ao plano horizontal. Para prevenir o contato visual com o braço preferido na fase de oclusão foi utilizado um anteparo opaco de madeira, que possuía uma parte inferior e outra vertical, ambas medindo 12 $\mathrm{cm}$. Na parte inferior do anteparo foi acoplado a uma haste de madeira, fazendo uma conexão com a haste lateral da cadeira, possibilitando o ajuste de posicionamento para cada bebê (Figura 1). 

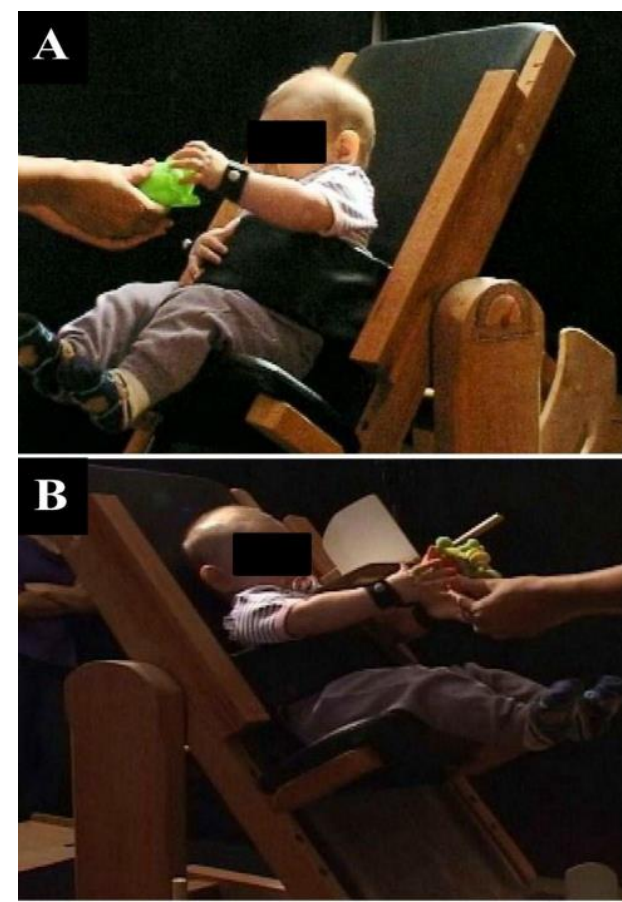

Figura 1. Imagens do arranjo experimental, mostrando o alcance em visão plena $(A)$ e com oclusão visual do braço esquerdo por anteparo (B).

Para realizar a análise cinemática dos movimentos do braço durante o alcance, foram fixados marcadores reflexivos ( $5 \mathrm{~mm}$ de diâmetro) em ambos os punhos, entre os processos estiloides da ulna e do rádio. $O$ alcance foi estimulado utilizando um de três brinquedos coloridos ( $5 \mathrm{~cm}$ de diâmetro), com formato e peso (aproximadamente $50 \mathrm{~g}$ ) similares. O brinquedo era posicionado no plano sagital mediano do corpo dos bebês, na altura dos ombros, e apresentado com as duas mãos pelo experimentador. A distância do brinquedo era correspondente ao comprimento dos braços dos bebês. Antes de iniciar o experimento, uma haste localizada em frente à cadeira foi usada como referência para manter fixa a posição de apresentação do brinquedo. $O$ alcance era considerado válido quando o bebê fixava o olhar no brinquedo e realizava o alcance com um ou com ambos os braços até tocá-lo. A fixação visual do bebê no brinquedo era monitorada visualmente pelo experimentador. Nas situações de desvio do olhar e interesse por outro objeto, em que o brinquedo era apresentado e o bebê não realizava o alcance, ou realizava o movimento sem fixar visualmente o objeto, a tentativa era repetida até que atingisse quatro alcances válidos. $O$ número máximo de tentativas repetidas foi 15 .
Os movimentos dos bebês foram filmados com quatro câmeras de vídeo digitais, sendo duas da marca JVC (modelo GY DV-300) e duas da marca Sony (DSR-PD170), com frequência de aquisição das imagens igual a $60 \mathrm{~Hz}$. As câmeras foram acopladas a tripés e mantidas à altura de $1 \mathrm{~m}$ em relação ao piso.

\section{Delineamento experimental e procedimentos}

Para início das atividades de coleta de dados foram fixados marcadores reflexivos em ambos os punhos. Em seguida, os bebês foram posicionados na cadeira infantil. $O$ experimento foi conduzido em três fases: visão plena préoclusão, oclusão visual do braço preferido e visão plena pós-oclusão. Na situação de visão plena pré-oclusão os bebês realizaram alcances sem qualquer restrição do campo visual, podendo visualizar os braços. O objetivo dessa fase foi identificar a preferência manual (maiores detalhes no subitem Análise dos Dados), em quatro alcances válidos. Para estimular os movimentos, um brinquedo era apresentado pelo experimentador no plano sagital mediano do corpo do bebê.

$\mathrm{Na}$ fase subsequente, os movimentos de alcance foram realizados com oclusão da visão do braço preferido, definido na fase de visão plena pré-oclusão. Os bebês tiveram a visão do braço preferido ocluída por meio do anteparo disposto na cadeira infantil. $O$ anteparo era colocado ao lado da cabeça, correspondente ao lado do braço preferido. Com esta disposição, prevenia-se que os bebês estabelecessem contato visual com o braço preferido durante toda a fase de oclusão, sem prejudicar os movimentos de alcance (ver Figura 1). Para dois bebês que apresentaram preferência manual indefinida um deles teve oclusão do braço direito e o outro teve a oclusão do braço esquerdo. A oclusão visual do braço preferido foi mantida durante toda a fase de oclusão visual. Esta fase foi realizada por 7 períodos de $60 \mathrm{~s}$, visando o aumento cumulativo da oclusão do braço. Durante os períodos de 60 $\mathrm{s}$, o movimento de alcance não era estimulado. Dessa forma, para que os bebês permanecessem na cadeira durante esses períodos, eles eram distraídos com brincadeiras que não estimulavam movimentos de alcance. Assim, os bebês tiveram períodos ininterruptos em que apenas o braço não-preferido era visível. Após cada período de oclusão, o brinquedo era apresentado quatro vezes na mesma posição da fase de pré-oclusão 
totalizando um total de 28 alcances válidos nessa fase. Este procedimento foi feito para verificar o efeito da oclusão cumulativa ao longo do tempo. Dois bebês necessitaram de intervalos de repouso de $2 \mathrm{~min}$. entre os períodos de oclusão para alimentação. Durante este intervalo os bebês podiam manter contato visual com o braço que havia sido ocluído e ao retornar à cadeira era repetido o último período de oclusão, dando prosseguimento ao experimento.

Na situação de visão plena pós-oclusão, os quatro alcances eram realizados com visão plena de ambos os braços, apresentando-se 0 brinquedo na mesma posição das condições anteriores. Esta situação experimental teve o propósito de verificar a persistência da preferência manual do braço que teve a visibilidade ocluída.

\section{Análise dos Dados}

A análise da preferência manual foi realizada por meio da frequência de alcances com o braço preferido em cada situação experimental. Foi utilizado o índice de preferência manual, dado pela seguinte equação: $I P=(D-E) /(D+E)$ (SOUZA; TUDELLA; TEIXEIRA, 2011), na qual $D$ e $E$ se referem à frequência absoluta de alcances realizados com os braços direito e esquerdo, respectivamente. Para alcances unimanuais foi atribuído um ponto para o braço que tocou 0 objeto. Nos casos de alcance bimanual foi atribuído um ponto para cada braço. A força da direção da preferência manual foi determinada por meio da média entre os índices observados em cada situação experimental. Quanto mais próximo de 1 , maior a preferência pelo braço direito; quanto mais próximo de -1 , maior a preferência pelo braço esquerdo; sendo 0.2 a -0.2 o escore correspondente à indefinição de preferência manual.

$\mathrm{Na}$ análise do desempenho motor, os movimentos foram reconstruídos tridimensionalmente empregando o Sistema Dvideowß 5.0 (BARROS et al., 1999). Para cálculo das variáveis de interesse foi utilizado o programa Matlab®. Para essa análise foram consideradas apenas as tentativas de alcance com o braço que foi ocluído nas três fases experimentais, com o objetivo de avaliar a influência da visibilidade sobre o desempenho motor desse braço. O início do alcance foi definido como o momento em que o bebê iniciava o movimento com um ou com ambos os braços para alcance do brinquedo, após ter fixado o olhar sobre ele. $O$ final do alcance foi definido como o momento do toque no brinquedo. $O$ alcance bimanual foi considerado quando ambos os braços eram direcionados simultaneamente ao alvo por pelo menos um quarto da trajetória.

O desempenho motor foi analisado por meio das seguintes variáveis: (a) tempo de movimento: intervalo de tempo entre o início e final do movimento (CARVALHO; TUDELLA; SAVELSBERGH, 2007); (b) índice de retidão do movimento: razão entre a distância da mão ao brinquedo no início da ação e a distância percorrida pela mão (CARVALHO; TUDELLA; SAVELSBERGH, 2007); (c) velocidade média: cálculo da razão entre a distância percorrida e a duração do movimento; (d) número de unidades de movimento: cada unidade de movimento é definida como um vale entre dois picos de velocidade; a magnitude mínima considerada foi maior do que $1 \mathrm{~cm} / \mathrm{s}$ - utilizada para quantificar os ajustes realizados durante a trajetória do movimento (THELEN; CORBETTA; SPENCER, 1996); (e) pico de velocidade: maior velocidade instantânea durante o alcance (CARVALHO; TUDELLA; SAVELSBERGH, 2007); e (f) tempo relativo de desaceleração: razão entre o tempo de pós-pico de velocidade até o toque no brinquedo e tempo de movimento - utilizada para verificar a duração da fase de correção do movimento de alcance. A amplitude de movimento foi usada como critério de exclusão de tentativas, selecionando-se apenas tentativas com amplitude mínima de $5 \mathrm{~cm}$.

A análise da preferência manual foi feita com estatística descritiva e por meio da prova de Friedman para comparações entre as fases, enquanto que a análise do desempenho motor foi realizada empregando-se um modelo de análise de variância de fator único para o efeito de teste (pré $\mathrm{x}$ oclusão $\mathrm{x}$ pós) para medidas repetidas. $\mathrm{O}$ nível de significância foi estabelecido em 5\%.

\section{Resultados}

\section{Preferência manual}

A análise descritiva da preferência manual na situação de visão plena pré-oclusão indicou que dois bebês apresentaram preferência manual direita forte $(100 \%$ de alcances com o braço direito), um bebê apresentou preferência manual 
direita fraca $(50 \%$ de alcances com o braço direito e $50 \%$ bimanuais), e dois apresentaram preferência manual indefinida ( $25 \%$ de alcances com o braço direito, $25 \%$ com o braço esquerdo e $50 \%$ bimanuais). Os valores de frequência de alcances, nas três fases experimentais, são apresentados na Tabela 1. Nota-se que na situação de oclusão visual do braço preferido os bebês com preferência manual direita forte reduziram a frequência de alcances com o braço ocluído em relação à fase de visão plena préoclusão para $37 \%$ das tentativas e aumentaram a frequência de alcances bimanuais. Na situação de visão plena pós-oclusão visual estes bebês apresentaram comportamentos diferentes.
Enquanto um deles realizou $100 \%$ dos alcances com braço que havia sido ocluído, retornando à preferência manual original, o outro realizou apenas $25 \%$ dos alcances com o braço originalmente preferido. $\mathrm{O}$ bebê com preferência direita fraca reduziu de $50 \%$ para $29 \%$ os movimentos de alcance com o braço preferido na situação de oclusão visual. Um dos efeitos mais evidentes da oclusão visual foi observado nos bebês com preferência manual indefinida. Os resultados mostraram que estes bebês não realizaram alcances com o braço não-visível durante a fase de oclusão, e que este comportamento foi mantido durante a fase pósoclusão.

Tabela 1. Porcentagens e frequências absolutas (entre parênteses) de alcances realizados com o braço direito $(D)$, esquerdo $(E)$ e bimanuais (bi) nas três fases experimentais.

\begin{tabular}{|c|c|c|c|c|c|c|c|c|c|}
\hline & \multicolumn{3}{|c|}{ Pré } & \multicolumn{3}{|c|}{ Oclusão } & \multicolumn{3}{|c|}{ Pós } \\
\hline & D & E & $\mathbf{B i}$ & D & E & $\mathrm{Bi}$ & D & E & $\mathbf{B i}$ \\
\hline $\mathrm{Da}$ & $\begin{array}{c}100 \\
(4)\end{array}$ & $\begin{array}{c}0 \\
(0)\end{array}$ & $\begin{array}{c}0 \\
(0)\end{array}$ & $\begin{array}{l}37 \\
(9)\end{array}$ & $\begin{array}{c}0 \\
(0)\end{array}$ & $\begin{array}{c}63 \\
(15)\end{array}$ & $\begin{array}{l}25 \\
(1)\end{array}$ & $\begin{array}{l}50 \\
\text { (2) }\end{array}$ & $\begin{array}{l}25 \\
(1)\end{array}$ \\
\hline $\mathrm{Ke}$ & $\begin{array}{c}100 \\
(4)\end{array}$ & $\begin{array}{c}0 \\
(0)\end{array}$ & $\begin{array}{c}0 \\
(0)\end{array}$ & $\begin{array}{c}37 \\
(10)\end{array}$ & $\begin{array}{l}15 \\
(4)\end{array}$ & $\begin{array}{c}48 \\
(13)\end{array}$ & $\begin{array}{c}100 \\
(4)\end{array}$ & $\begin{array}{c}0 \\
(0)\end{array}$ & $\begin{array}{c}0 \\
(0)\end{array}$ \\
\hline Is & $\begin{array}{l}50 \\
(2)\end{array}$ & $\begin{array}{c}0 \\
(0)\end{array}$ & $\begin{array}{l}50 \\
(2)\end{array}$ & $\begin{array}{c}29 \\
(10)\end{array}$ & $\begin{array}{l}11 \\
(4)\end{array}$ & $\begin{array}{c}60 \\
(21)\end{array}$ & $\begin{array}{c}100 \\
(4)\end{array}$ & $\begin{array}{c}0 \\
(0)\end{array}$ & $\begin{array}{c}0 \\
(0)\end{array}$ \\
\hline Dv & $\begin{array}{l}25 \\
(1)\end{array}$ & $\begin{array}{l}25 \\
(1)\end{array}$ & $\begin{array}{l}50 \\
(2)\end{array}$ & $\begin{array}{c}0 \\
(0)\end{array}$ & $\begin{array}{l}100 \\
(28)\end{array}$ & $\begin{array}{c}0 \\
(0)\end{array}$ & $\begin{array}{c}0 \\
(0)\end{array}$ & $\begin{array}{c}100 \\
(4)\end{array}$ & $\begin{array}{c}0 \\
(0)\end{array}$ \\
\hline Sa & $\begin{array}{l}25 \\
(1)\end{array}$ & $\begin{array}{l}25 \\
(1)\end{array}$ & $\begin{array}{l}50 \\
(2)\end{array}$ & $\begin{array}{c}86 \\
(24)\end{array}$ & $\begin{array}{c}0 \\
(0)\end{array}$ & $\begin{array}{l}14 \\
(4)\end{array}$ & $\begin{array}{c}100 \\
(4)\end{array}$ & $\begin{array}{c}0 \\
(0)\end{array}$ & $\begin{array}{c}0 \\
(0)\end{array}$ \\
\hline
\end{tabular}

Nota: Da e Ke: preferência direita forte, Is: preferência direita fraca, Dv e Sa: preferência manual indefinida - Dv: oclusão do braço direito e Sa: oclusão do braço esquerdo.

$\mathrm{Na}$ Figura 2 é apresentada a frequência relativa dos movimentos de alcance do braço nãovisível no decorrer das sessões de oclusão visual. Os dados revelaram que os bebês nas três categorias de preferência tenderam a reduzir ao longo do tempo a frequência de uso do braço não-visível durante a fase de oclusão. Este efeito foi mais marcante nos bebês com preferência indefinida, com realização de alcances com o braço não-visível apenas na primeira sessão de oclusão visual por um dos bebês, enquanto que 0 outro não executou qualquer alcance com o braço não-visível. Para os demais bebês o efeito foi progressivo, com o braço não-visível sendo usado menos nas últimas sessões de oclusão em comparação com as primeiras sessões. $O$ bebê com preferência direita fraca foi a única exceção, com aumento da frequência de uso do braço preferido na fase pós-oclusão.

A análise inferencial comparou os índices de preferência manual para os valores de préoclusão, última sessão de oclusão, representando o tempo final da fase de oclusão, e pós-oclusão. Os resultados indicaram efeito significante, $\chi^{2}(2$, $\mathrm{N}=5)=6,71, p<0,05$. Comparações post hoc entre as fases por meio da prova de Wilcoxon indicaram diferença significante entre as fases pré-oclusão e oclusão, $Z(5)=2,02, p<0,05$, mas não nas demais comparações (valores de $Z<$ 1,61). 


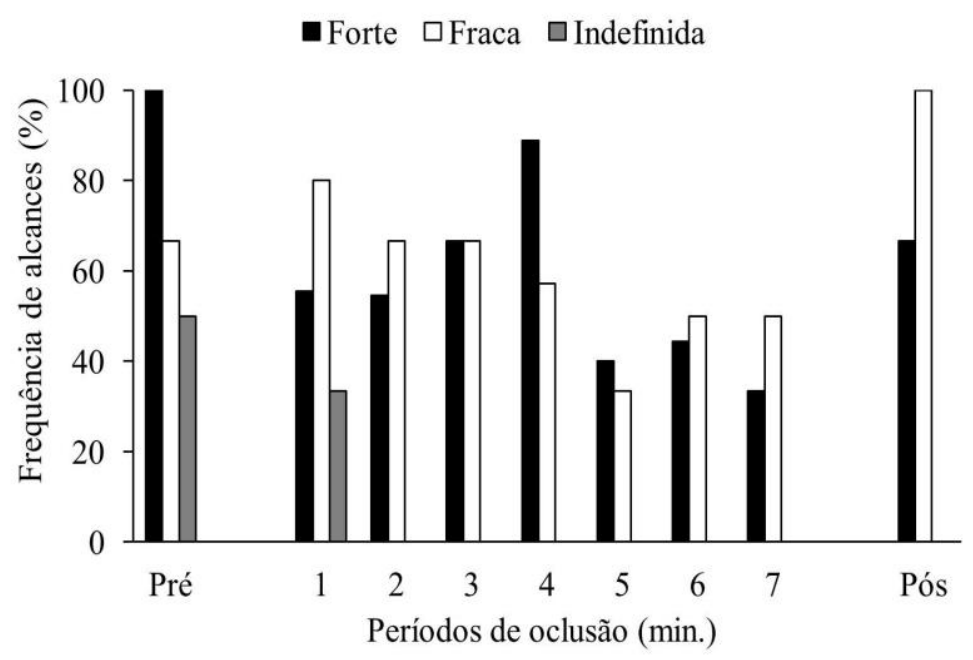

Figura 2. Frequências relativas de movimentos de alcance com o braço que teve visibilidade ocluída em função dos períodos de oclusão (min.), para bebês com preferência manual direita forte, direita fraca e indefinida.

Tabela 2. Médias e desvios padrão (entre parênteses) dos valores de cada variável cinemática de alcances com o braço que teve visibilidade ocluída em função da fase experimental, com os respectivos valores de $F$ e $p$.

\begin{tabular}{lccccc}
\hline & Pré & Oclusão & Pós & $\boldsymbol{F}$ & $\boldsymbol{p}$ \\
\cline { 2 - 6 } Tempo de movimento (s) & 0,83 & 1,07 & 0,90 & 1,60 & 0,30 \\
& $(0,04)$ & $(0,22)$ & $(0,41)$ & & \\
Pico de velocidade (cm/s) & 23,50 & 31,46 & 41,47 & 2,19 & 0,22 \\
Velocidade média (cm/s) & $(6,14)$ & $(5,44)$ & $(14,26)$ & & \\
& 12,69 & 14,23 & 21,25 & 1,18 & 0,39 \\
Índice de retidão & $(3,07)$ & $(2,27)$ & $(11,20)$ & & \\
& 0,75 & 0,69 & 0,65 & 0,91 & 0,46 \\
Tempo de desaceleração (\%) & $(0,08)$ & $(0,08)$ & $(0,14)$ & & \\
& 62,62 & 53,63 & 59,06 & 0,02 & 0,97 \\
Unidades de movimento $(\mathbf{n})$ & $(16,02)$ & $(10,09)$ & $(25,03)$ & & \\
& 4,00 & 5,94 & 4,33 & 1,94 & 0,25 \\
\hline
\end{tabular}

\section{Desempenho motor}

Os valores médios para as variáveis cinemáticas são apresentados na Tabela 2. Os resultados revelaram ausência de diferenças significantes para as variáveis, indicando que os bebês apresentaram desempenho motor semelhante nas situações experimentais de préoclusão, oclusão e pós-oclusão visual.

\section{Discussão}

O presente estudo teve como propósito investigar o efeito da oclusão visual de um dos braços sobre sua frequência de uso e desempenho motor em bebês aos cinco meses de idade. Os resultados demonstraram que a oclusão visual de um dos braços reduziu temporariamente sua frequência de alcances. Tal efeito foi observado mesmo em bebês com preferência manual definida, à medida que a oclusão promoveu redução na frequência de alcances unimanuais com o braço ocluído e aumentou os alcances bimanuais. Com a retirada do anteparo de oclusão, os alcances unimanuais com o braço que havia sido ocluído tornaram-se mais frequentes, indicando um efeito temporário da oclusão visual sobre esse braço. Nos bebês com preferência manual indefinida $o$ efeito da oclusão visual foi mais notório, uma vez que eles fizeram uso quase exclusivo do braço que foi mantido visível durante a situação de oclusão, deixando de usar o braço que teve visibilidade ocluída mesmo após a retirada da oclusão visual. A análise cinemática indicou que a variação no uso dos braços em função da oclusão visual não 
foi acompanhada por variação de desempenho motor, visto que não houve diferenças significativas nas variáveis cinemáticas entre as situações com visão plena versus oclusão visual.

\section{Desempenho motor}

Os resultados da análise cinemática indicando semelhança entre os movimentos realizados em cada fase experimental indicam que a oclusão da visibilidade do braço não levou a prejuízos marcantes no desempenho motor. Considerandose que o tempo de movimento, índice de retidão e número de unidades de movimento têm sido utilizadas como algumas das principais variáveis para caracterizar o desempenho motor do alcance (cf. CORBETTA; THELEN, 1999; SAINBURG, 2002; SAINBURG; KALAKANIS, 2000; THELEN et al., 1996), fica aparente que o alcançar voluntário não é guiado predominantemente por informação visual aos cinco meses de idade. Em experimentos em que bebês foram desprovidos da visão do movimento de alcance (CLIFTON; PERRIS; BULLINGER, 1991; MCCARTY; ASHMEAD, 1999), foi observada redução no tempo de movimento, número de unidades de movimento e índice de retidão ao executarem o alcance, indicando alteração no modo de controle na ausência da visão. Esses comportamentos distintos podem ter sido devidos aos diferentes ambientes em que foi realizada a tarefa. Nos estudos de Clifton et al. (1991) e de McCarty e Ashmead (1999) os bebês executaram alcances em quartos completamente escuros, dificultando também a localização do alvo por meio da informação visual. Nestes casos o prejuízo de controle motor pode ter se dado não pela falta de aferência visual do braço em movimento, mas pela falta de visibilidade do alvo para preensão. Diferentemente destes estudos prévios, em nosso experimento foi permitido aos bebês utilizarem informação visual para localizar a posição do objeto, o que pode ter facilitado o planejamento do alcance com o braço ocluído a partir de informação proprioceptiva. A ausência de prejuízo significante no desempenho em variáveis cinemáticas pela oclusão visual do braço de alcance sugere que aos cinco meses a regulação de movimentos voluntários não é feita de forma importante por meio de feedback visual on-line. É possível, assim, que os movimentos sejam controlados por meio de ajustes antecipatórios ou por ajustes de um movimento para outro. Ajustes antecipatórios ou realizados entre movimentos têm sido documentados em indivíduos adultos (ver KHAN et al., 2006, para revisão), mas pouca informação está disponível sobre o potencial de uso destas formas de controle em bebês. Desta forma, os resultados aqui apresentados oferecem dados de interesse a respeito do efetivo uso de informação visual para realização de ajustes visuomotores em idades precoces.

\section{Preferência manual}

A análise dos resultados de preferência manual confirmou a hipótese de que a oclusão visual do braço preferido leva a uma redução da frequência de alcances unimanuais com esse braço durante a fase de oclusão, induzindo maior frequência de alcances bimanuais ou unimanuais com o braço visível. Um dos pontos de maior interesse nestes resultados foi que mesmo bebês demonstrando forte preferência pelo braço direito tiveram redução notável na sua frequência de uso durante a fase de oclusão. Um destes bebês pareceu ter sua preferência manual afetada pela oclusão visual do braço preferido também na fase pós-oclusão, uma vez que passou a manifestar preferência manual esquerda. $O$ efeito mais evidente de oclusão visual, contudo, foi observado nos bebês com preferência manual indefinida. Nestes bebês a falta de contato visual extinguiu os movimentos com o braço não-visível na segunda sessão de oclusão, e depois deste momento nenhum outro movimento com o braço contrário foi feito para alcançar os brinquedos. $\mathrm{O}$ bebê que teve a visibilidade do braço direito ocluída passou a realizar todos os movimentos com o braço esquerdo, enquanto que o bebê com oclusão visual do braço esquerdo passou a fazer todos os movimentos com o braço direito a partir da segunda sessão de oclusão. Um aspecto adicional a ser destacado é que este perfil de preferência manual formado durante a fase de oclusão tendeu a permanecer na fase subsequente, na qual ambos os braços estavam visíveis. Aparentemente, o contato visual com um dos braços é um importante fator na seleção daquele segmento corporal para a realização do alcançar. Estes resultados são consistentes com observações preliminares na situação de visão plena pré-oclusão, mostrando que bebês com preferência manual direita fraca são mais propensos a modificar sua preferência manual (cf. LECONTE; FAGARD, 2006). No entanto, tal efeito não foi encontrado de forma evidente em bebês com preferência manual definida. A divergência entre resultados prévios e aqueles aqui apresentados pode ser devida à maior força do 
efeito visual pela abordagem experimental empregada em nosso estudo.

Conforme o bebê focaliza um dos braços e observa seus movimentos mobiliza recursos atencionais (CONTE et al., 2007), podendo favorecer sua escolha em ações subsequentes (MICHEL, 1981). À medida que a visão é reconhecida como um importante elemento na captura da atenção para o planejamento e orientação do movimento (SONG; NAKAYAMA, 2006; VON HOFSTEN, 1982), é plausível que a variação da preferência manual em bebês pela falta de visão de um dos braços esteja associada à mobilização de recursos atencionais. No entanto, a maioria dos estudos com bebês aborda tal proposta de maneira descritiva (DUBOWITZ et al., 1986; VAN DER MEER, 1997; VAN DER MEER; VAN DER WEEL; LEE, 1996; VON HOFSTEN, 1982; VON HOFSTEN; FAZELZANDY, 1984). A este respeito, a análise da ativação de áreas cerebrais em função do foco atencional tem sido bastante utilizada em estudos com adultos (BINKOFSKI et al., 2002; JOHANSEN-BERG; MATTHEWS, 2002; JUEPTNER et al. 1997). De acordo com estes estudos, a atenção quando orientada para os movimentos das mãos aumenta a atividade das áreas corticais relacionadas à organização do movimento, incrementando a força de conexão entre os córtices pré-frontal dorsal e pré-motor. Por outro lado, a falta de foco atencional para a ação tende a diminuir a conectividade entre estas áreas corticais (ROWE et al., 2002). Estes achados permitem supor que a visualização de um dos braços é capaz de capturar automaticamente recursos atencionais dos bebês, podendo favorecer a seleção daquele segmento corporal para realizar ações motoras.

Os resultados aqui relatados são consistentes com a noção de que a orientação da face em supino para o lado direito em idades precoces, favorecendo $\circ$ contato visual com o braço ipsilateral, é um fator capaz de modular a preferência manual durante a infância (CORYELL; MICHEL, 1978; GAZZANIGA, 1971; MICHEL, 1981; MICHEL; HARKINS, 1986; OCKLENBURG et al., 2010). A demonstração em nossos resultados de que a preferência manual é afetada pelo contato visual e de que a alteração na frequência de uso das mãos é persistente para um período subsequente sugere que 0 contato visual com um dos braços é um potente elemento no desenvolvimento da preferência manual na infância. A maleabilidade de modificação da preferência manual em curto intervalo de tempo, como observado aqui e em estudos longitudinais (CORBETTA; THELEN, 1999; CORBETTA; THELEN, 2002), sugere que experiências visuomanuais no período de emergência do alcançar podem ser críticas para 0 desenvolvimento da lateralidade nas idades subsequentes.

\section{Comentários conclusivos}

Alguns aspectos importantes para o desenvolvimento da lateralidade infantil emergem das observações aqui relatadas. Na dimensão teórica, ficou evidente o caráter dinâmico da preferência manual, com alterações na frequência de uso dos braços com apenas poucos minutos de oclusão de visibilidade do braço preferido. Como corolário, foi suportada a proposição de que o maior contato visual com o braço direito nos primeiros meses de vida, em função da orientação lateral predominante da face, pode favorecer o desenvolvimento da preferência manual direita. Nesse sentido, o desenvolvimento da preferência manual poderia ser induzido por fatores experienciais ao invés de determinado pela herança filogenética, como proposto por Annett (1985). Na dimensão aplicada, tornou-se aparente a importância de favorecer a visibilidade de um segmento corporal quando se deseja induzir o uso em uma série de tentativas de prática em bebês. Este fator poderia ter um papel relevante particularmente nos primeiros meses de vida, em atividades orientadas à promoção do desenvolvimento motor especificamente de um segmento corporal com movimentos prejudicados por disfunção neural ou de outra natureza.

\section{Referências}

ANNETT, M. Left, right, hand and brain: The right shift theory. Hillsdale, NJ: Erlbaum. 1985.

ASHTON, G. C. Handedness: an alternative hypothesis. Behavioral Genetics, New York, NY: Elsevier, v.12, n. 2, p. 125-147. 1982.

BARROS, R. M. L.; BRENZIKOFER, R.; LEITE, N. J.; FIGUEROA, P. J. Development and evaluation of a system for three-dimensional kinematic analysis of human movements. Journal of Biomedical Engineering, London, UK, v. 15, p. 79-86. 1999.

BINKOFSKI, F.; FINK, G. R.; GEYER, S.; BUCCINO, G.; GRUBER, O.; SHAH, N. J., et al. Neural activity in human primary motor cortex 
areas $4 a$ and $4 p$ is modulated differentially by attention to action. Journal of Neurophysiology, Bethesda, MD, USA, v. 88, p. 514-519. 2002.

BRYDEN, P. J.; MAYER, M.; ROY, E. A. Influences of task complexity, object location, and object type on hand selection in reaching in left and right-handed children and adults.

Developmental Psychobiology, New Orleans, LA: Wiley Online Library, v. 53, n. 1, p. 47-58. 2011.

CARVALHO, R. P.; TUDELLA, E.;

SAVELSBERGH, G. J. P. Spatio-temporal parameters in infant's reaching movements are influenced by body orientation. Infant Behavior \& Development, Amsterdam, NL: Elsevier Science Publishing Co, v. 30, p. 26-35. 2007.

CLIFTON, R. K.; PERRIS, E. E.; BULLINGER, A. Infants' perception of auditory space.

Developmental Psychology, Washington, DC: American Psychological Association, v. 27, p. 187197. 1991.

CONTE, A.; GILIO, F.; IEZZI, E.; FRASCA, V.; INGHILLERI, M.; BERARDELLI, A. Attention influences the excitability of cortical motor areas in healthy humans. Experimental Brain Research, New York, NY: Elsevier, v. 182, p.109-117. 2007.

CORBETTA, D.; GUAN, Y.; WILLIAMS, J. L. Infant eye-tracking in the context of goal-directed actions. Infancy, New York, NY: Elsevier, p. 1-24. 2011.

CORBETTA, D.; THELEN, E. Lateral biases and fluctuations in infants' spontaneous arm movements and reaching. Developmental Psychobiology, New Orleans, LA: Wiley Online Library, v. 34, p. 237-255. 1999.

CORBETTA, D.; THELEN, E. Behavioral fluctuations and the development of manual asymmetries in infancy: Contribution of the dynamic systems approach. In S. J. Segalowitz \& I. Rapin (Eds.), Handbook of Neuropsychology: Child Neuropsychology (v. 8, p. 309-329). Amsterdam, The Netherlands: Elsevier Science Publishing Co. 2002.

CORBETTA, D.; WILLIAMS, J.; SNAPP-CHILDS, W. Plasticity in the development of handedness: Evidence from normal development and early asymmetric brain injury. Developmental Psychobiology, New Orleans, LA: Wiley Online Library, v. 48, p. 460-471. 2006.

CORYELL, J. F.; MICHEL, G. F. How supine postural preferences of infants can contribute toward the development of handedness. Infant Behavior \& Development, Amsterdam, NL:
Elsevier Science Publishing Co, v. 1, p. 245-257. 1978.

DARLING, W. G.; RIZZO, M. Developmental lesions of visual cortex influence control of reaching. Neuropsychology, Washington, DC: American Psychological Association, v. 39, p. 346351. 2001.

DUBOWITZ, L. M.; MUSHIN, J.; DE VRIES, L.; ARDEN, G. B. Visual function in the newborn infant: Is it cortically mediated? Lancet, Philadelphia, PA: Elsevier Inc., v. 1, p. 1139-1141. 1986.

GAZZANIGA, M. S. Changing hemisphere dominance by changing reward probability in splitbrain monkeys. Experimental Neurology, New York, NY: Elsevier, v. 33, n. 2, p. 412-419. 1971.

HARKINS, D. A.; MICHEL, G. F. Evidence for a maternal effect on infant hand-use preferences. Developmental Psychobiology, Malden, MA: Wiley Online Library, v. 21, p. 535-541. 1988.

HARKINS, D. A.; UZGIRIS, I. C. Hand-use matching between mothers and infants during the first year. Infant Behavior \& Development, Amsterdam, NL: Elsevier Science Publishing Co, v. 14, p. 289-298. 1991.

JOHANSEN-BERG, H.; MATTHEWS, P. M. Attention to movement modulates activity in sensori-motor areas, including primary motor cortex. Experimental Brain Research, New York, NY: Elsevier, v. 142, p. 13-24. 2002.

JUEPTNER, M.; STEPHEN, K. M.; FRITH, C. D.; BROOKS, D. J.; PASSINGHAM, R. E. Anatomy of motor learning. Journal of Neurophysiology, Bethesda, MD, USA, v. 77, p. 1313-1324. 1997.

KHAN, M. A.; FRANKS, I. M.; ELLIOTT, D.; LAWRENCE, G. P.; CHUA, R.; BERNIER, P. M., et al. Inferring online and offline processing of visual feedback in target-directed movements from kinematic data. Neuroscience \& Biobehavioral Reviews, New York, NY: Elsevier, v. 30, n. 8, p. 1106-1121. 2006.

LASKY, R. The effect of visual feedback of the hand on the reaching and retrieval behavior of young infants. Child Development, Malden, MA: Wiley Online Library, v. 48, p. 112-117. 1977.

LECONTE, P.; FAGARD, J. Which factors affect hand selection in children's grasping in hemispace? Combined effects of task demand and motor dominance. Brain and Cognition, New York, NY: Elsevier, v. 60, p. 88-93. 2006. 
LEVY, J.; NAGYLAKI, T. A model for the genetics of handedness. Genetics, Bethesda, MD, USA, v. 72, p. 117-128. 1972.

MANNS, M. The epigenetic control of asymmetry formation: Lessons from the Avian visual system. In Y. Malashichev \& A. W. Deckel (Eds.),

Behavioural and morphological asymmetries in vertebrates. (p. 13-23). Georgetown: Landes Bioscience. 2006.

MCCARTY, M. E.; ASHMEAD, D. H. Visual control of reaching and grasping in infants. Developmental Psychobiology, Malden, MA: Wiley Online Library, v. 35, n. 3, p. 620-631. 1999.

MCDONNELL, P. M. Patterns of eye-hand coordination in the first year of life. Canadian Journal of Psychology, Washington, DC: American Psychological Association, v. 33, p. 253267. 1979.

MCMANUS, I. C. Handedness, language dominance and aphasia: A genetic model. Psychological Medicine-Monograph Supplement, Cambridge: Cambridge University Press, v. 8, p. 1-40. 1985.

MICHEL, G. F. Right-handedness: A consequence of infant supine head-orientation preference? Science, v. 212, p. 685-687. 1981.

MICHEL, G. F.; HARKINS, D. A. Postural and lateral asymmetries in the ontogeny of handedness during infancy. Developmental Psychobiology, Malden, MA: Wiley Online Library, v. 19, p. 247-258. 1986.

NUDO, R. J.; MILLIKEN, G. W.; JENKINS, W. M.; MERZENICH, M. M. Use-dependent alterations of movement representations in primary motor cortex of adult squirrel monkeys. Journal of Neuroscience, Washington, DC: American Psychological Association, v. 16, p. 785-807. 1996.

OCKLENBURG, S.; BÜRGER, C.; WESTERMANN, C.; SCHNEIDER, D.; BIEDERMANN, H.; GÜNTÜRKÜN, O. Visual experience affects handedness. Behavioural Brain Research, New York, NY: Elsevier, v. 207, p. 447-451. 2010.

PROVINS, K. A. Handedness and speech: A critical reappraisal of the role of genetic and environmental factors in the cerebral lateralization of function. Psychological Review, Washington, DC: American Psychological Association, v. 104, p. 554-571. 1997.

RIDDING, M. C.; FLAVEL, S. C. Induction of plasticity in the dominant and non-dominant motor cortices of humans. Experimental Brain
Research, New York, NY: Elsevier, v. 171, p. 551557. 2006.

ROSANDER, K.; NYSTRÖM, P.; GREDEBÄCK, G.; VON HOFSTEN, C. Cortical processing of visual motion in young infants. Vision Research, Oxford, v. 47, n. 12, p. 1614-1623. 2007.

ROWE, J.; FRISTON, K.; FRACKOWIAK, R.; PASSINGHAM, R. Attention to action: Specific modulation of corticocortical interactions in humans. Neuroimage, New York, NY: Elsevier, v. 17, n.2, p. 988-998. 2002

SAINBURG, R. L. Evidence for a dynamicdominance hypothesis of handedness.

Experimental Brain Research, New York, NY: Elsevier, v. 142, n. 2, p. 241-258. 2002.

SAINBURG, R. L.; KALAKANIS, D. Differences in control of limb dynamics during dominant and nondominant arm reaching. Journal of Neurophysiology, Bethesda, MD, USA, v. 83, n. 5, p. 2661-2675. 2000.

SONG, J. H.; NAKAYAMA, K. Role of focal attention on latencies and trajectories of visually guided manual pointing. Journal of Vision, Rockville, MD, USA, v. 6, p. 982-995. 2006.

SOUZA, R. M.; TUDELLA, E.; TEIXEIRA, L. A. Preferência manual na ação de alcançar em bebês em função da localização espacial do alvo. Psicologia: Reflexão e Crítica, Porto Alegre, RS: UFRS, v. 24, n. 2, p. 318-325. 2011.

TEIXEIRA, L. A. Timing and force components in bilateral transfer of learning. Brain and

Cognition, New York, NY: Elsevier, v. 44, p. 455465. 2000.

TEIXEIRA, L. A. Assimetrias laterais. Controle motor (p. 298-340). 1. Ed. Barueri, SP: Manole. 2006.

THELEN, E.; CORBETTA, D.; SPENCER, J. Development of reaching during the first year: Role of movement speed. Journal of Experimental Psychology: Human Perception and Performance, Washington, DC: American Psychological Association, v. 22, p. 1059-1076. 1996.

TURKEWITZ, G.; BIRCH, H.; MOREAU, T. Head position and receptor organization in the human neonate. Journal of Experimental Child

Psychology, New York, NY: Elsevier, v. 4, p. 169177. 1966.

TURKEWITZ, G., MOREAU, T., DAVIS, L., \& $\mathrm{BIRCH}, \mathrm{H}$. Factors affecting lateral differentiation in the human neonate. Journal of Experimental 
Child Psychology, New York, NY: Elsevier, v. 8, p. 483-493. 1969.

VAN DER MEER, A. L. Keeping the arm in the limelight: Advanced visual control of arm movements in neonates. European Journal of Paediatric Neurology, New York, NY: Elsevier, v. 4, p. 103-108. 1997.

VAN DER MEER, A. L.; VAN DER WEEL, D. N. The functional significance of arm movements in neonates. Science, v. 267, n.5198, p. 693-695. 1995.

VAN DER MEER, A. L. H.; VAN DER WEEL, F. R.; LEE, D. N. Lifting weights in neonates: Developing visual control of reaching. Scandinavian Journal of Psychology, Malden, MA: Wiley Online Library, v. 37, p. 424-436. 1996.

VERVERS, I. A. P.; VRIES, J. I. P.; VAN GEIJN, H. P.; HOPKINS, B. Prenatal head position from 12-38 weeks. Early Human Development, New York, NY: Elsevier, v. 39, n. 2, p. 83-91. 1994.

VIVIANI, J.; TURKEWITZ, G.; KARP, E. A relationship between laterality of functioning at 2 days and at 7 years of age. Bulletin of the Psychonomic Society, v. 12, n. 3, p. 189-192. 1978.

VON HOFSTEN, C. Eye-hand coordination in the newborn. Developmental Psychobiology, Malden, MA: Wiley Online Library, v. 18, n. 3, p. 450-461. 1982.

VON HOFSTEN, C.; FAZEL-ZANDY, S.

Development of visually guided hand orientation in reaching. Journal of Experimental Child

Psychology, New York, NY: Elsevier, v. 38, n. 2, p. 208-219. 1984.
Agradecimentos: Lívia Silveira Pogetti e Rosana Machado de Souza foram suportadas com bolsa de estudos provida pela FAPESP (processos no 2009/ 15193-3 e 2010/01581-9, respectivamente). Luis Teixeira e Eloísa Tudella foram suportados com bolsa produtividade pelo CNPq (processos no 303052/2010-4 e 306444/2009-7, respectivamente).

Endereço:

Lívia Silveira Pogetti

Rua Izabel Iliza de Lima, 491 Bairro: São Joaquim Franca SP Brasil

14.406-36

e-mail: liviapogetti@hotmail.com

Recebido em: 21 de março de 2012. Aceito em: 10 de fevereiro de 2013.

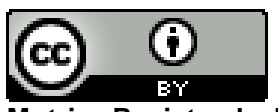

Motriz. Revista de Educação Física. UNESP, Rio Claro, SP, Brasil - elSSN: 1980-6574 - está licenciada sob Creative Commons - Atribuicão 3.0 